\title{
AFM lithography for fabrication of magnetic nanostructures and devices
}

\author{
Yasushi Takemura $^{\mathrm{a}, *}$ and Jun-ichi Shirakashi ${ }^{\mathrm{b}}$ \\ ${ }^{a}$ Yokohama National University, 79-5 Tokiwadai, Hodogaya, Yokohama 240-8501, Japan \\ ${ }^{\mathrm{b}}$ Tokyo University of Agriculture and Technology, 2-24-16 Naka-cho, Koganei, Tokyo 184-8588, Japan
}

Elsevier use only: Received date here; revised date here; accepted date here

\begin{abstract}
Nanostructures of oxide were fabricated on ferromagnetic thin films by a nano-oxidation technique using an atomic force microscope. Electrical and magnetic properties in the magnetic nanostructures were directly modified by the nano-oxidation. A ferromagnetic single electron transistor exhibited a single electron tunneling due to the Coulomb blockade effect. A direct modification of magnetic domain structures was also achieved by this nano-lithography technique.
\end{abstract}

Keywords: magnetic nanostructure, atomic force microscopy, nanolithography.

\section{Introduction}

When a negative bias voltage is applied to a tip of a scanning probe microscope, nanostructures of oxide can be obtained on surfaces of conductive thin films [1]. This is an electrochemical reaction between the conductive thin films and water on their surfaces. As this anodic oxidation process does not require any pretreatments such as a resist coating, it is an attractive technique for fabricating devices with a nanometer scale. Fabrications of nanostructures based on ferromagnetic thin films by the AFM nanooxidation have been demonstrated [2]. A planar-type magnetic tunnel junction (MTJ) is one of the most attractive device structures that can be fabricated by this nano-lithography technique. As its junction area can be smaller than that of a conventional multi-layer type MTJ, an observation of a single electron tunneling due to the Coulomb blockade effect is expected. In this paper, recent progress in direct modifications of electrical and magnetic properties of magnetic nanostructures and devices by the AFM nano-oxidation is reported.

\section{Experiments}

Figure 1 illustrates a schematic view of the nano-

* Corresponding author. Tel. \& Fax: +81-45-339-4151.

E-mail address: takemura@ynu.ac.jp. 


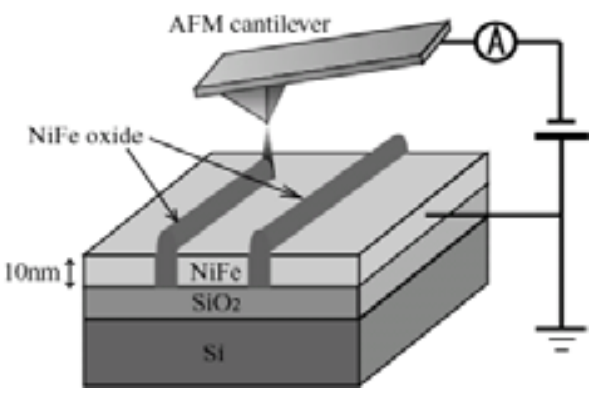

Fig. 1. Schematic of AFM nano-oxidation technique.

oxidation technique with using an atomic force microscope (AFM). When a negative bias voltage is applied to the conductive AFM cantilever positioned at a surface of a NiFe thin film, nanostructures of NiFe-oxide are fabricated by an electrochemical reaction between the $\mathrm{NiFe}$ and water in air. In this study, the $\mathrm{Si}_{3} \mathrm{~N}_{4}$ cantilever coated with Au was used. Current mapping images recorded by the AFM have indicated that the nanostructures of oxide fabricated by this technique exhibit insulating properties [3].

A size of the fabricated nanostructures of oxide depended on an applied voltage to the conductive cantilever, which could be explained by introducing a threshold of an electric field for promoting the oxidation. The threshold electric field of the order of $10^{7} \mathrm{~V} / \mathrm{m}$ was deduced by fitting experimental results to calculated values [3]. It was also indicated that a curvature radius of the cantilever tip determined a minimum size of the nano-oxide. It is useful to measure a current passing through the cantilever during the nano-oxidation. This current is called a faradaic current. A volume of the fabricated nanooxide can be theoretically calculated by integrating the faradaic current with time. Recently, Kuramochi et al. reported that the current was successfully measured and that volumes of fabricated nanodots of oxide agreed with integrated current values [4]. The faradaic current was also measured and analyzed during fabrication of nanowires of oxide based on $\mathrm{Si}$, GaAs substrates and metallic films [5]. It was noticed that both of measured currents and sizes of the fabricated nano-oxides strongly depended on conditions of surfaces, e.g. Si substrate with a hydrogen-passivated surface or capped with a native oxide on the surface.

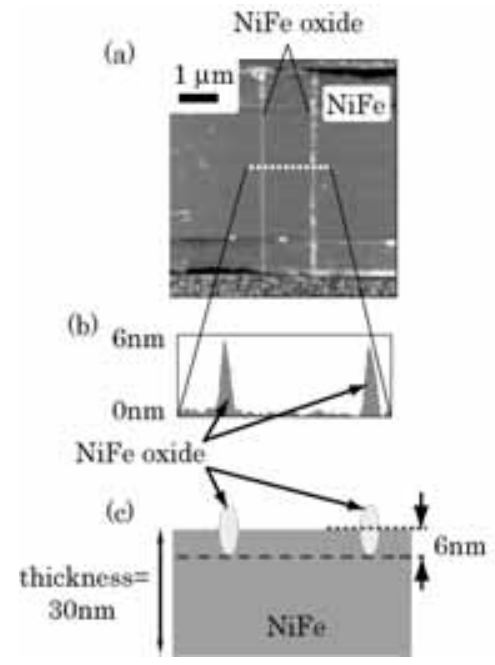

Fig. 2. AFM image of patterned NiFe thin film with two nanowires of NiFe oxide fabricated by AFM nano-oxidation (a). The height profile (b) and schematic of the cross section (c) are also indicated. The film thickness of the sample was $30 \mathrm{~nm}$, whereas the depth of the oxide was $6 \mathrm{~nm}$.

\section{Results and Discussion}

The AFM nano-oxidation can be used for fabricating magnetic devices with a nanometer scale. Figure 2(a) shows an AFM image of a patterned NiFe thin film of $4 \mathrm{~mm}$ width and $30 \mathrm{~nm}$ thickness. Two nanowires of NiFe oxide were fabricated as to cross the patterned stripe of the NiFe thin film. A height profile was traced along a white dotted line indicated in Fig. 2(a). The height of the nano-oxide wires was about $6 \mathrm{~nm}$ as observed from the profile shown in Fig. 2(b). Oxides fabricated by the AFM nano-oxidation from GaAs and Si can be selectively etched by a wet process using chemical solutions. After removing the nano-oxides of $\mathrm{Si}$ and GaAs, AFM images can determine their depth burrowing into GaAs or Si. It has been found that a height and a depth of each nanostructure of oxide are almost equivalent from experimental results using GaAs and Si. Therefore, it can be assumed that a depth of the nano-wires of NiFe oxides is approximately $6 \mathrm{~nm}$ as illustrated by a cross section in Fig. 2(c). When a depth of fabricated oxide does not reach to a bottom of a conductive film, the film exhibits a metallic current-voltage 
characteristic. Anisotropic magnetoresistance (AMR) was observed from the patterned NiFe sample with the nanowires of oxide shown in Fig. 2. The AMR curve of the sample plotted as a function of an applied magnetic field did not change from that of a NiFe thin film without a surface modification of nano-oxides. When the oxidized depth was larger than $40 \%$ of the film thickness, a pinning of domain wall propagation during a magnetization reversal was observed in the AMR curve of the NiFe thin film with nanowires of oxide [6].

In case that a fabricated oxide burrows down to a bottom of a film, a planar-type metal/insulator/metal tunnel junction is obtained. An observation of rectifying current-voltage characteristics is expected in electrical measurements of this tunnel device. Figure 3(a) illustrates a schematic of the planar-type double MTJ. The AFM nano-oxidation was applied to a patterned NiFe thin film of $2 \mu \mathrm{m}$ width and $4 \mathrm{~nm}$ thickness in order to fabricate two nanowires of oxide and also to reduce the channel width. As a result, an isolated island of NiFe can be formed. Figure 3(b) shows an AFM image of the NiFe patterned film with its narrowed channel and the isolated island. A dimension of the NiFe island in this sample was 100 $\times 100 \mathrm{~nm}^{2}$. This is a basic structure of a single electron device. An enhancement of a tunnel magnetoresistance (TMR) due to a single electron tunneling by the Coulomb blockade effect has been studied both of theoretically [7] and experimentally $[8,9]$. In order to observe the Coulomb blockade effect, a junction area of the MTJ should be nanometer scale. Comparing with a conventional multi-layer type tunnel junction, the planar-type

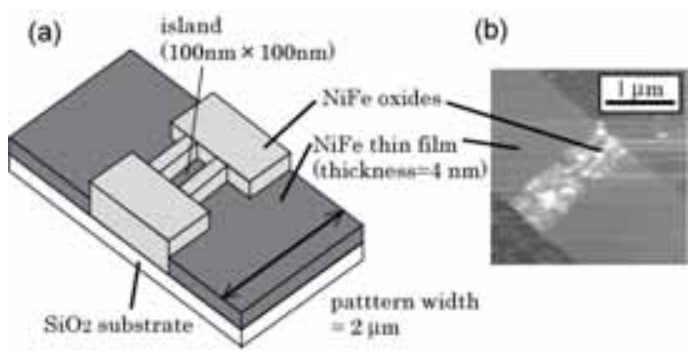

Fig. 3. Schematic of NiFe-based double tunnel junction with small isolated island of $\mathrm{NiFe}$ (a) and AFM image of the fabricated sample structure. tunnel junction has an advantage in its smaller junction area that is attributed to the island electrode [10].

Rectifying current-voltage characteristics have been observed in electrical measurements of $\mathrm{Ni}$-, NiFe- and CoFe-based planar-type tunnel junctions. Details on the fabrication and the current-voltage characteristics have been reported in ref. 10 and 11 . In this paper, electrical properties of a ferromagnetic single electron transistor (FMSET) are discussed. A fabricated sample consisted a patterned Ni film of 3 nm thickness, whose channel width was constricted by the AFM nano-oxidation. A dimension of the isolated island of $\mathrm{Ni}$ was of $30 \times 30 \mathrm{~nm}^{2}$. A gate electrode was also prepared in order to bias the tunnel junction [10]. Figure 4 shows a drain current-drain voltage characteristic of the $\mathrm{Ni} / \mathrm{Ni}$ oxide/Ni-based FMSET measured at $17 \mathrm{~K}$ with a gate bias voltage of $0 \mathrm{~V}$. Asymmetric bias conditions were used in the electrical measurements. As shown in the figure, the drain current was well suppressed at around $0 \mathrm{~V}$, and the Coulomb blockade regime could be observed with the corresponding charging energy of $20 \mathrm{meV}$. A dependence of the drain current on the gate voltage was also measured. Figure 5 shows a drain currentgate voltage characteristic of the FMSET at $17 \mathrm{~K}$ with the drain bias voltage of $5 \mathrm{mV}$. Although the background current depending on the gate voltage appeared, well-modulated periodic oscillation of the drain current with $70 \mathrm{mV}$ periodicity could be observed. Total capacitance of the FMSET is

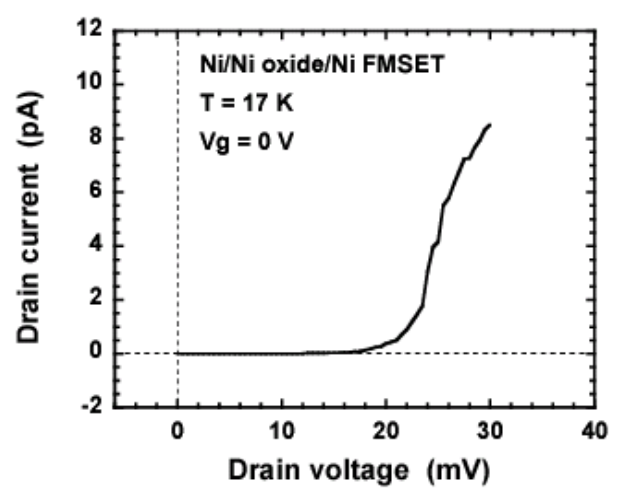

Fig. 4. Drain current-drain voltage characteristic of $\mathrm{Ni} / \mathrm{Ni}$ oxide based ferromagnetic single electron transistor measured at $17 \mathrm{~K}$. 


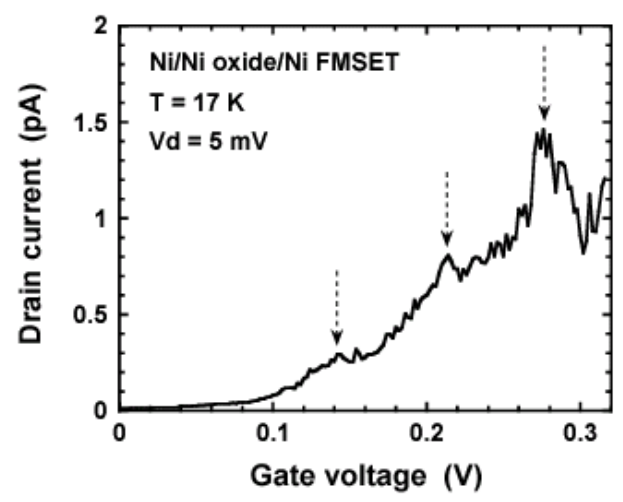

Fig. 5. Drain current-gate voltage characteristic of Ni/Ni oxide based ferromagnetic single electron transistor measured at $17 \mathrm{~K}$.

deduced to be $4 \mathrm{aF}$ from the observed charging energy. The island dimension is calculated to be $15 \times$ $15 \mathrm{~nm}^{2}$ if the total capacitance of the device is mainly given by the self capacitance of the island. A difference in the dimension between the actual $(30 \times$ $\left.30 \mathrm{~nm}^{2}\right)$ and the calculated $\left(15 \times 15 \mathrm{~nm}^{2}\right)$ values is presumably imputed to other capacitances e.g. junction capacitances.

Control of magnetic properties in magnetic nanostructures by the AFM nano-oxidation has been also studied. It was found that magnetic domain structures in patterned Co rectangles could be modified [12]. As the magnetic domain was divided by the nanowire of oxide, it was proposed that a magnetic anisotropy in magnetic nanostructures could be directly controlled by a nanolithography technique using the AFM.

\section{Conclusions}

A recent progress in the AFM nano-oxidation of ferromagnetic thin films is reported. As a modification of electrical properties of the films by the nano-oxidation, planar-type tunnel junctions were fabricated based on patterned $\mathrm{NiFe}, \mathrm{Ni}$ and other magnetic thin films. A channel width of the junctions could be constricted into as narrow as $100 \mathrm{~nm}$ or less also by the AFM nano-oxidation technique. A ferromagnetic single electron transistor, which had a small isolated island, exhibited a single electron tunneling due to the Coulomb blockade effect. In order to utilize spin devices, control of magnetic domains in ferromagnetic electrodes is significant. A direct modification of magnetic domain structures by the AFM nano-oxidation was also achieved. These results suggest that the AFM nano-oxidation is an attractive lithography technique for fabricating the ferromagnetic materials and devices with nanometer scale.

\section{References}

[1] J. A. Dagata, J. Schneir, H. H. Harary, C. J. Evans, M. T. Postek, and J. Bennett, Appl. Phys. Lett. 56 (1990) 2001.

[2] Y. Takemura and J. Shirakashi, Jpn. J. Appl. Phys., 39 (2000) L1292.

[3] Y. Takemura, S. Kidaka, K. Watanabe, Y. Nasu, T. Yamada and J. Shirakashi, J. Appl. Phys. 93 (2003) 7346.

[4] H. Kuramochi, F. Pérez-Murano, J. A. Dagata and H. Yokoyama, Nanotechnology 15 (2004) 297.

[5] N. Funakoshi, G. Watanabe, T. Yamada, Y. Takemura and J. Shirakashi, 13th Int. Conf. Scanning Tunneling Microscopy/Spectroscopy and Related Techniques (STM'05), Sapporo, Japan, 2005.

[6] G. Watanabe, S. Koizumi, K. Watanabe, T. Yamada, Y. Takemura and J. Shirakashi, 1st Int. Conf. One-dimensional Nanomaterials, Taipei, Taiwan, 2005.

[7] S. Takahashi and S. Maekawa, Phys. Rev. Lett. 80 (1998) 1758.

[8] K. Ono, H. Shimada and Y. Ootuka, J. Phys. Soc. Jpn. 66 (1997) 1261.

[9] S. Mitani, S. Takahashi, K. Takanashi, K. Yakushiji, S. Maekawa and H. Fujimori, Phys. Rev. Lett. 81 (1998) 2799.

[10] J. Shirakashi and Y. Takemura, IEEE Trans. Magn. 40 (2004) 2640.

[11] J. Shirakashi and Y. Takemura, J. Magn. Magn. Mater. 272276 (2004) 1581.

[12] Y. Takemura, S. Hayashi, F. Okazaki, T. Yamada and J. Shirakashi, Jpn. J. Appl. Phys. 44 (2005) L285. 